\title{
Catalytic creation of a bubble universe induced by quintessence in five dimensions
}

\author{
Issei Koga ${ }^{1}$ and Yutaka Ookouchi ${ }^{2,1, *}$ \\ ${ }^{1}$ Department of Physics, Kyushu University, Fukuoka 810-8581, Japan \\ ${ }^{2}$ Faculty of Arts and Science, Kyushu University, Fukuoka 819-0395, Japan
}

(Received 1 September 2021; accepted 29 November 2021; published 16 December 2021)

\begin{abstract}
We investigate the bubble nucleation in five-dimensional spacetime catalyzed by quintessence. We especially focus on the decay of a metastable Minkowski vacuum to an anti-de Sitter vacuum and study the dynamics of the bubble on which a four-dimensional expanding universe is realized. We also discuss the trans-Planckian censorship conjecture and impose a constraint on the parameter space of the catalysis. As an application of this model, we propose an inflation mechanism and an origin of the dark energy in the context of quintessence in five dimensions.
\end{abstract}

DOI: 10.1103/PhysRevD.104.126015

\section{INTRODUCTION}

The structure of vacua in unified theories has attracted wide attention recently. Especially, after the discovery of the Higgs particle and precise measurements of top quark mass, it have been believed that our Universe is metastable even within the standard model [1]. This possibility was first pointed out by [2-4]. Related work on this instability of our Universe has been done from various points of view [5]. In string theories, the vacuum structure becomes more involved, which is known as the string landscape (see [6] for example), and has been discussed recently in the context of swampland conjectures [7]. One of the remarkable conjectures is the de Sitter conjecture [8], which prohibits making a four-dimensional de Sitter space by compactifying the internal space. See [9] for reviews and references therein. This conjecture is controversial and still under debate. However, it would be interesting to explore other realizations of our Universe in string theories in light of the conjectures. An interesting avenue was opened up by the authors of [10], in which they realized a four-dimensional universe on a bubble in five dimensions created by a decay process of a metastable anti-de Sitter (AdS) vacuum. The radiation and matter in four dimensions are realized in terms of a black hole and a string cloud in five dimensions. Naïvely, since the bubble is the boundary of two AdS spaces, the fourdimensional gravity can be localized on the bubble in the same spirit as Randall-Sundrum [11] scenario (see [12,13]

\footnotetext{
* Corresponding author. yutaka.ookouchi@artsci.kyushu-u.ac.jp

Published by the American Physical Society under the terms of the Creative Commons Attribution 4.0 International license. Further distribution of this work must maintain attribution to the author(s) and the published article's title, journal citation, and DOI. Funded by SCOAP .
}

for more recent studies on this issue). The catalytic effects caused by the string cloud and the black hole in this context was recently discussed in [14] and showed that the catalysis provides a kind of the selection rule to the cosmological constant on the bubble universe. This paper can be regarded as a continuation of this study, and we try to engineer the inflation sector and the dark energy in this context.

Catalytic effects in field theories were first pointed out by [15] and discussed in various contexts such as realistic model building [16] and decay processes in stringy theories [17]. Also, this idea has been discussed in the context of gravitational theories [18] initiated by [19]. In this paper, we study vacuum decay along the lines of these papers, especially by using the method developed in [18] to treat a singular bounce solution. Recently, catalytic effects in gravitational theories have been discussed in various contexts [20-25].

In this paper, we introduce quintessence in this scenario and discuss catalysis induced by it. A discontinuity of quintessence on the bubble can be interpreted as a fourdimensional quintessence. One of the remarkable features of the quintessence is the time dependence of $w_{(4)}$ in the state equation. Even if $w_{(4)} \simeq-1$ at the present age of the Universe, it could be larger at the early stage. It would be interesting if quintessence can play a role of catalyst when the bubble universe is created. Moreover, we will use the quintessence to engineer the inflation at the early stage and the dark energy at the late stage of the Universe.

The organization of this paper is as follows: in Sec. II, we review black hole solutions spherically surrounded by quintessence in four and five dimensions. Also we briefly review junction conditions for connecting two solutions with different parameters. Then, we show how to compute the bounce action for the decay of metastable vacuum and discuss a recent development on the bounce action for a solution with singularities along the lines of $[3,18,21]$. 
In Sec. III, we show catalytic effects induced by quintessence for the decay process of a metastable Minkowski vacuum to an AdS vacuum. In Sec. IV, we consider a model including two types of quintessence. First, we study catalytic effects induced by the quintessence and show a selection rule of bubbles in five dimensions. Then, we further impose a constraint by using the trans-Planckian censorship conjecture [26]. After that, we discuss an application of this model to a realization of inflation and the dark energy on the bubble universe. We use the freezing-type quintessence as an inflaton and thawing-type as the dark energy at the present age. Section V is devoted to conclusions and summary. In Appendix A, we quickly review the Coleman-de Luccia (CDL) bounce action in five dimensions $[4,14]$. Throughout this paper, we assume the parameter range $-1 \leq w \leq 0$ for a quintessential field. However, there is an interesting field for acceleration of the Universe, namely the phantom field with $w<-1$. In the Appendix B, we show the catalytic effect induced by the phantom field.

\section{GENERAL ARGUMENTS}

In this paper, we incorporate inflation and the dark energy with the bubble Universe realized in five dimensions [10]. Toward this goal, we use quintessence as candidates for inflaton and the dark energy. We treat spherically symmetric gravitational solutions for quintessence and study junctions of two solutions with different parameters. Hence, we first review the solutions in four and five dimensions, then we show basic formulas that will be used in computing the bounce action in the next section. To compute the bounce action, by using the method developed by Coleman [3], we solve the equation of motion for the bubble, which is the junction surface separating two regions, in Euclideanized theory and plug the solution back into the action. We also comment that the singularity at the origin of the solutions does not contribute to the bounce action.

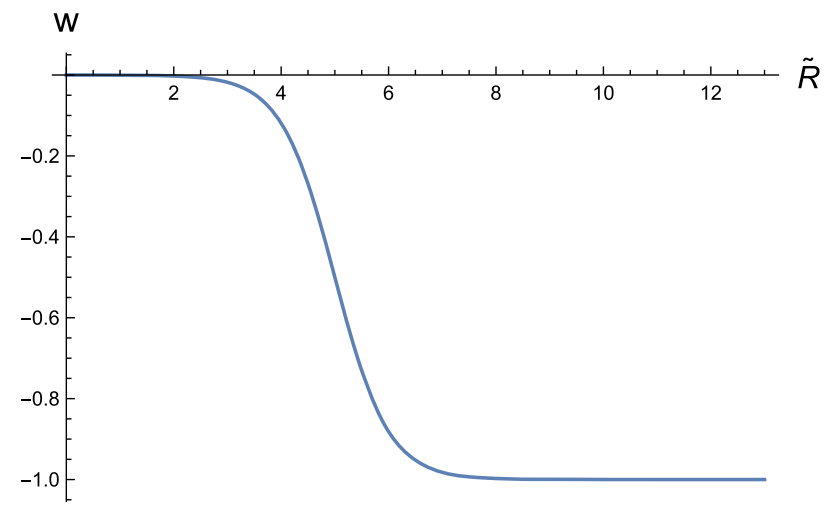

\section{A. Gravitational solution for quintessence}

Here, we quickly review the solutions for quintessence in four and five dimensions along the lines of [27,28]. First, we treat four dimensions. In the standard cosmology, the state equation relates the pressure $p$ with the energy density $\rho ; p=w_{(4)} \rho$. To distinguish from five-dimensional quintessence, we add the subscript. In four dimensions, the cosmological constant, radiation, and matter correspond to $w_{(4)}=-1,1 / 3,0$, respectively. We use the terminology "quintessence" in a broad sense in which all the states except these three cases are quintessence. Moreover, quintessence states can be divided into two parts by the acceleration of the universe. From the Friedmann equation,

$\frac{\ddot{a}}{a}=-\frac{4}{3} G_{4}\left(1+3 w_{(4)}\right) \rho \quad($ in four dimensions $)$,

we see that when $w_{(4)} \leq-1 / 3$, the Universe is accelerated by quintessence while $w_{(4)}>-1 / 3$ corresponds to deceleration.

Time dependence of $w_{(4)}$ varies from model to model and various kinds of phenomenological models for quintessence has been proposed. See [29] for reviews. Among them, the freezing and thawing models are suitable for our purpose. So we discuss them by idealizing the dependence of $w_{(4)}$ as functions shown in Fig. 1 for the sake of simplicity. In the freezing model, $w_{(4)}$ starts around zero. It gradually gets smaller and eventually reaches -1 . In the thawing model, it starts around -1 and finally becomes zero. Note that Fig. 1 is just a schematic picture to demonstrate our assumption, so the numbers in the figure do not have any sense. Throughout this paper, we simply assume the scale factor dependence of $w_{(4)}$ as in Fig. 1 without specifying explicit models.

Black hole solutions surrounded by quintessence were shown in [27]

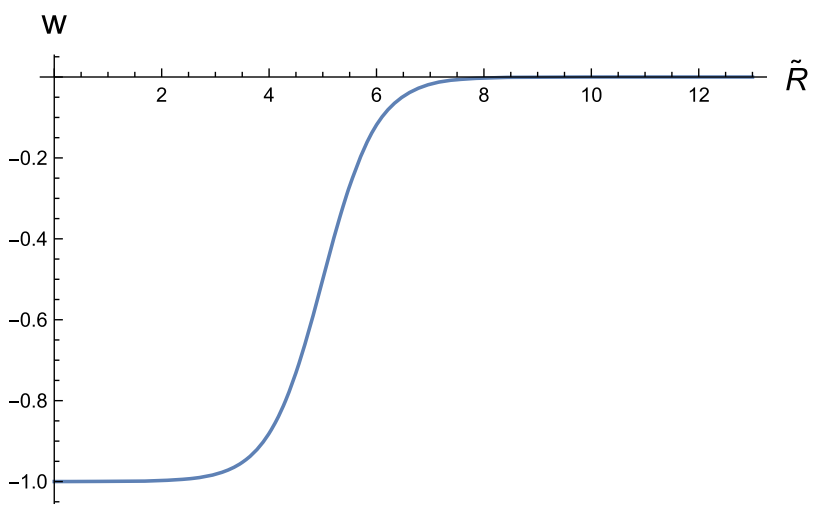

FIG. 1. Schematic picture of $w$. We assume that $w$ in five dimensions varies as a function of scale factor. In the freezing model, it starts around 0 and goes to -1 , while in the thawing, it starts from -1 and goes to 0 . In Secs. III and IV, we discuss the four-dimensional universe realized on the bubble created in five dimensions. In this case, $\tilde{R}$, which is a normalized scale factor (2.13), corresponds to the size of the bubble in five dimensions. 


$$
d s^{2}=-f_{K}(r) d t^{2}+f_{K}^{-1}(r) d r^{2}+r^{2} d \Omega_{2}^{2},
$$

where $d \Omega_{2}^{2}$ is the two-dimensional round metric and

$$
f_{K}(r)=1-\frac{r_{g}^{2}}{r^{2}}-\sum_{n}\left(\frac{r_{n}}{r}\right)^{3 w_{(4)}^{(n)}+1} .
$$

The label $n$ runs from 1 to the total number of quintessence. Although the solutions corresponding to the accelerated universe have both cosmological and event horizons, the solutions for $-1 / 3<w_{(4)}<0$, there is no cosmological horizon.

These quintessential solutions were extended to higher dimensions [28]. Here, let us focus on five dimensions for our purpose. In this case, the solution is given by

$$
d s^{2}=-f(r) d t^{2}+\frac{d r^{2}}{f(r)}+r^{2} d \Omega_{3}^{2},
$$

where $d \Omega_{3}^{2}$ is the three-dimensional round metric and the function $f(r)$ is

$$
f(r)=1-\frac{r_{\mathrm{BH}}^{2}}{r^{2}}-\frac{\Lambda^{(5)}}{6} r^{2}-\sum_{n} \frac{q^{(n)}}{r^{4 w^{(n)}+2}} .
$$

Here, we wrote the contribution of the cosmological constant separately, because we will deal with models having both cosmological constant and quintessence later. In five dimensions, the accelerated universe corresponds to $w<-1 / 2$.

In the solutions of (2.2) and (2.4), contributions from black holes exist. These are important in making spherically symmetric quintessence in a realistic situation. However, in the discussions below, we put $r_{\mathrm{BH}}=0$ for the sake of simplicity and illustrate the catalytic effect induced by quintessence. As we will see in Secs. III and IV, inhomogeneity of these solutions, which can be seen in the singularity at the origin, enhances the decay rate of the metastable vacuum.

\section{B. Junction condition and equation of motion for bubble}

In discussing the bubble nucleation, we study a junction surface of two different spherically symmetric solutions. Over the surface, there are discontinuities of physical quantities such as the curvature and the energy-momentum tensor. The discontinuity of the curvature can be expressed in terms of the extrinsic curvature $K_{i j}$ as follows: the Einstein equation on the surface can be reduced to

$$
K_{i j}^{+}-K_{i j}^{-}=8 \pi G_{5}\left(S_{i j}-\frac{1}{3} \gamma_{i j} S\right),
$$

where $i, j$ runs from 1 to the dimension of the bubble. This is known as Israel's junction condition [30]. Hereafter, we write the subscript $+(-)$ for quantities outside (inside) the bubble. For the sake of simplicity, we impose the thin-wall approximation and use $S_{i j}=-\sigma \gamma_{i j}$. With this notation and by taking the trace of the extrinsic curvature, the junction condition becomes

$$
K^{+}-K^{-}=\frac{32 \pi G_{5}}{3} \sigma,
$$

where we used $S=-4 \sigma$.

For later convenience, let us modify the expression and make the condition a bit simpler form. We basically adopt the same notations used in [21] and define $\eta=\bar{\sigma} l, \bar{\sigma}=$ $4 \pi G_{5} \sigma / 3$ and

$$
l^{2}=\frac{6}{\Delta \Lambda^{(5)}}, \quad \gamma=\frac{4 \bar{\sigma} l^{2}}{1+4 \bar{\sigma}^{2} l^{2}}, \quad \alpha^{2}=1+\frac{\Lambda_{-}^{(5)} \gamma^{2}}{6}
$$

where $\Delta \Lambda^{(5)}=\Lambda_{+}^{(5)}-\Lambda_{-}^{(5)}$. Two geometries with different parameters are connected at $r=R(\lambda)(\lambda$ is the proper time on the bubble), thus the induced metric on the bubble becomes the Friedmann type form,

$$
d s^{2}=-d \lambda^{2}+R^{2}(\lambda) d \Omega_{3}^{2} .
$$

To estimate the decay rate for this bubble nucleation, by following the Coleman's method [3], we introduce the Euclidean time defined by $t=-i \tau$ and look for a classical solution in the Euclideanized theory. By computing the extrinsic curvature with the notations above, (2.7) can be written as

$$
\frac{1}{R}\left(f_{+} \dot{\tau}_{+}-f_{-} \dot{\tau}_{-}\right)=-\frac{8 \pi G_{5}}{3} \sigma
$$

where $\dot{\tau}_{ \pm}$satisfy the following relations

$$
f_{ \pm} \dot{\tau}_{ \pm}^{2}+\frac{\dot{R}^{2}}{f_{ \pm}}=1
$$

Using these expressions and introducing $\bar{f}=\left(f_{+}+f_{-}\right) / 2$ and $\Delta f=f_{+}-f_{-}$, we obtain the equation of motion for the bubble

$$
\dot{R}^{2}=-\bar{\sigma}^{2} R^{2}+\bar{f}-\frac{(\Delta f)^{2}}{16 \bar{\sigma}^{2} R^{2}} .
$$

For numerical calculations, we define dimensionless coordinates as in [21],

$$
\tilde{R}=\frac{\alpha R}{\gamma}, \quad \tilde{\lambda}=\frac{\alpha \lambda}{\gamma}, \quad \tilde{\tau}=\frac{\alpha \tau}{\gamma} .
$$

It is also convenient to introduce dimensionless parameters for quintessence, 


$$
Q_{ \pm(n)}^{(w)}=\left(\frac{\alpha}{\gamma}\right)^{4 w+2} q_{ \pm(n)},
$$

and to express the functions $f_{ \pm}$in terms of them

$$
f_{ \pm}(\tilde{R})=1+\left(\frac{\gamma}{l_{ \pm} \alpha}\right)^{2} \tilde{R}^{2}-\sum_{n} \frac{Q_{ \pm(n)}^{(w)}}{\tilde{R}^{4 w^{(n)}+2}} .
$$

Throughout this paper, since we assume vacuum decay between AdS (or Minkowski) spacetimes in five dimensions, we define the typical length of the spacetimes as

$$
l_{ \pm}^{2}=-\frac{6}{\Lambda_{ \pm}^{(5)}}
$$

The equation of motion for the radius of the bubble can be expressed as

$$
\begin{aligned}
(\dot{\tilde{R}})^{2}= & 1-\tilde{R}^{2}-\sum_{n}\left(\bar{Q}_{(n)}+\frac{\Delta Q_{(n)}}{8 \eta^{2}}\right) \frac{1}{\tilde{R}^{4 w^{(n)}+2}} \\
& -\frac{1}{16 \eta^{2}}\left(\frac{l \alpha}{\gamma}\right)^{2}\left(\sum_{n} \frac{\Delta Q_{(n)}}{\tilde{R}^{4 w^{(n)}+3}}\right)^{2}
\end{aligned}
$$

where we introduced

$\bar{Q}_{(n)}=\frac{Q_{+(n)}^{(w)}+Q_{-(n)}^{(w)}}{2}, \quad \Delta Q_{(n)}=Q_{+(n)}^{(w)}-Q_{-(n)}^{(w)}$.

Now, let us discuss the cosmological constant for the four-dimensional bubble universe. By comparing with the Friedmann equation, we can read off it from the second term on the right-hand side of (2.17) in dimensionful coordinates (note that this equation is expressed by Euclidean time),

$$
\frac{\alpha^{2}}{\gamma^{2}} l^{2}=\eta^{2}-\frac{1+\delta^{2}}{2\left(1-\delta^{2}\right)}+\frac{1}{16 \eta^{2}}=\frac{\Lambda^{(4)} l^{2}}{3},
$$

where we defined $\delta=l_{-} / l_{+}$. The minus sign in the second term of (2.19), originating from background of the anti-de Sitter space, is important. If we assume de Sitter spaces in five dimensions for both sides of the bubble, then the sign becomes plus, which make it impossible to take the cosmological constant to be zero. From Fig. 2, we see that the cosmological constant on the bubble depends on the tension $\eta$ and in a wide range of parameter spaces it is positive. However, note that, as we will see below, not all the tension can be realized under the bubble nucleation. For the fixed value of catalyst, there is the minimum allowed value of $\eta$, below which there is not a bounce solution for the decay (one can check that by numerical computations for explicit models). When $\Lambda^{(4)}<0$, one can also explicitly check that there is no bounce solution

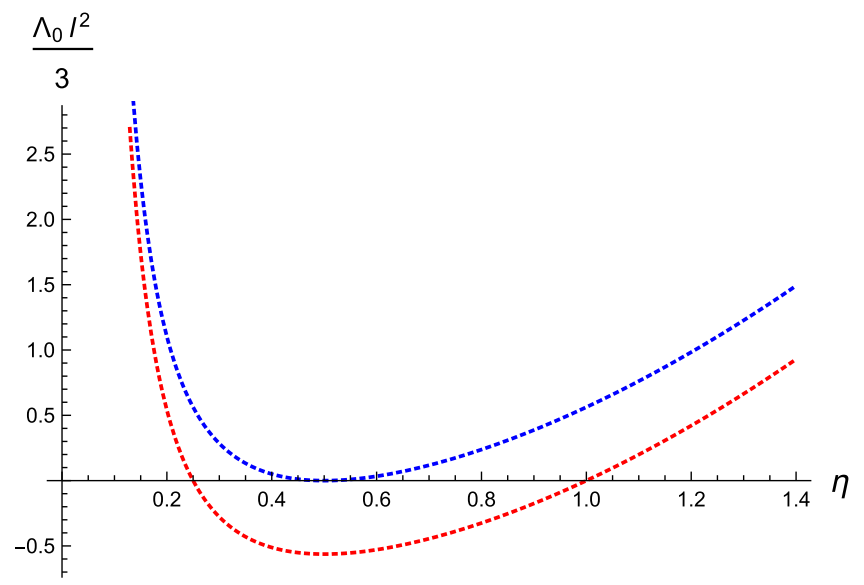

FIG. 2. The cosmological constant on the bubble as a function of the tension $\eta$. The blue and red curves correspond to $\delta=$ 0 and $6 / 10$.

for Eq. (2.17) in the most of $\eta$ (there might be a very small allowed window around the critical value of $\eta$ ). Hence, the four-dimensional AdS space cannot be created by this catalysis. So the minimum cosmological constant obtained by balancing the tension of the bubble and background AdS radius is $\Lambda^{(4)}=0$. In this case, the tension has to satisfy the following relation,

$$
\bar{\sigma}_{\mathrm{cr}}^{2}-\frac{1}{2}\left(\frac{1}{l_{+}^{2}}+\frac{1}{l_{-}^{2}}\right)+\frac{1}{16 \bar{\sigma}_{\mathrm{cr}}^{2}}\left(\frac{1}{l_{+}^{2}}-\frac{1}{l_{-}^{2}}\right)^{2}=0 .
$$

We call the bubble satisfying this condition as the critical bubble and denote its tension $\bar{\sigma}_{\mathrm{cr}}$. By solving this equation, we obtain the critical value of the tension as

$$
\bar{\sigma}_{\mathrm{cr}}=\frac{1}{2}\left(\frac{1}{l_{-}}-\frac{1}{l_{+}}\right), \quad \eta_{\mathrm{cr}}=\frac{1}{2} \sqrt{\frac{l_{+}-l_{-}}{l_{+}+l_{-}}} .
$$

Adopting the same notation in [10], let us introduce a parameter $\epsilon$ representing deviation from the critical value,

$$
\bar{\sigma}=\bar{\sigma}_{\mathrm{cr}}(1-\epsilon),
$$

and approximate the right-hand side of (2.19) a few order in $\epsilon$

$$
\Lambda^{(4)} \simeq \frac{6 \epsilon}{l_{+} l_{-}}+3 \epsilon^{2}\left(\frac{1}{l_{-}^{2}}+\frac{1}{l_{+}^{2}}+\frac{1}{l_{-} l_{+}}\right)
$$

Interestingly, by taking $l_{+} \rightarrow \infty$ (Minkowski limit), the leading order of the cosmological constant becomes zero. Hence, to make fine-tuning a bit mild, we assume a metastable Minkowski vacuum for the original geometry and study its decay process to AdS vacuum. It is worthy noting that if we naïvely take Minkowski vacua for both sides of the bubble, which corresponds to $l_{ \pm} \rightarrow \infty$, the 
parameters become $\alpha=1$ and $\gamma=\bar{\sigma}$, thus the cosmological constant given by the scale of the tension is $\Lambda^{(4)}=3 \bar{\sigma}^{2}$. In this case, $G_{4}$ and $\Lambda^{(4)}$ are roughly expressed by the same scale, namely the Planck scale, which cannot be acceptable in phenomenological point of view. In this scene, we assume AdS (or Minkowski for outside) spacetimes for both sides and study bubbles with tension near the critical value. Also, to make the four-dimensional Newton constant finite, we define $G_{4}=G_{5} / l$.

The quintessence in five dimensions can be interpreted as the one in four dimensions on the bubble. For example, a contribution to the Friedmann equation can be given by $1 / \tilde{R}^{4 w+4}$ in five-dimensional terminology. This should correspond to $1 / \tilde{R}^{3 w_{(4)}+3}$ in four dimensions. Thus, we get

$$
w_{(4)} \Leftrightarrow \frac{1}{3}(4 w+1) .
$$

We find that quintessence parameter $w$ in five dimensions corresponds to the states on the bubble as follows ${ }^{1}$ :

$$
w=\left\{\begin{array}{l}
-1 \quad \text { cosmological constant } \\
-\frac{1}{4} \text { matter } \\
0 \quad \text { radiation }
\end{array}\right. \text { (onthebubble) }
$$

In this ways, parameter range $-1 \leq w \leq 0$ covers all the states in four dimensions. Thus, below we consider only this parameter range for $w$ in five dimensions. ${ }^{2}$

\section{Calculation of the bounce action}

In this subsection, we quickly review how to compute the bounce action. Recently, Gregory, Moss and Withers showed how to treat singularities on bounce solutions [18]. Here, we outline the formulas without showing the details. To extract the contributions from the singularities, let us decompose the spacetime into two parts. Suppose that there are several singularities on the solution labeled by $i$. We denote the neighborhood of the singularities $\mathcal{B}=\sum_{i} \mathcal{B}_{i}$ and whole spacetime $\mathcal{M}$. Subtracting the singular parts, we obtain $\mathcal{M}-\mathcal{B}$. In the same way, the action can be decomposed into two parts, $I=I_{\mathcal{M}-\mathcal{B}}+I_{\mathcal{B}}$. Let us consider the first contribution

$I_{\mathcal{M}-\mathcal{B}}=-\frac{1}{16 \pi G_{5}} \int_{\mathcal{M}-\mathcal{B}} R-\int_{\mathcal{M}-\mathcal{B}} \mathcal{L}_{m}+\frac{1}{8 \pi G_{5}} \int_{\partial(\mathcal{M}-\mathcal{B})} K$.

This can be further divided into three parts, namely the contributions from in and outside of the bubble (we denote $\mathcal{W})$ and bubble itself,

\footnotetext{
${ }^{1}$ In four dimensions, $w_{(4)}=-1,0,1 / 3$ corresponds to the cosmological constant, matter, and radiation, respectively.

${ }^{2}$ In Appendix B, we discuss a phantom field with $w<-1$, which is not excluded from observations and study its catalytic effect.
}

$$
I_{\mathcal{M}-\mathcal{B}}=I_{-}+I_{+}+I_{\mathcal{W}}
$$

The action on the wall can be given by

$$
I_{\mathcal{W}}=-\int_{\mathcal{W}} \mathcal{L}_{m}=\int_{\mathcal{W}} \sigma
$$

Also, the curvature tensor of five dimensions can be decomposed in terms of the four dimensional curvature as follows:

$R={ }^{(5)} R-K^{2}+K_{i j}^{2}-2 \nabla_{i}\left(u^{i} \nabla_{j} u^{j}\right)+2 \nabla_{j}\left(u^{i} \nabla_{i} u^{j}\right)$,

where $u^{j}$ is the derivative of coordinates with respect to the proper time on the bubble. By exploiting these expressions, the action can be written as

$$
I_{ \pm}=-\frac{1}{8 \pi G_{5}} \int_{\mathcal{W}} K_{ \pm}+\frac{1}{8 \pi G_{5}} \int_{\mathcal{W}} n_{ \pm j} u^{i} \nabla_{i} u^{j}
$$

where $n^{\mu}$ is the normal vector perpendicular to the bubble and satisfies the condition, $1=g_{\mu \nu} n^{\mu} n^{\nu}$. Hence, the action (2.26) can be expressed as

$$
\begin{aligned}
I_{\mathcal{M}-\mathcal{B}} & =\int_{\mathcal{W}} \sigma-\frac{4}{3} \int_{\mathcal{W}} \sigma-\frac{1}{16 \pi G_{5}} \int_{\mathcal{W}}\left(f_{+}^{\prime} \dot{\tau}_{+}-f_{-}^{\prime} \dot{\tau}_{-}\right), \\
& =-\frac{1}{3} \int_{\mathcal{W}} \sigma-\frac{1}{16 \pi G_{5}} \int_{\mathcal{W}}\left(f_{+}^{\prime} \dot{\tau}_{+}-f_{-}^{\prime} \dot{\tau}_{-}\right) .
\end{aligned}
$$

Next, we show contributions to the bounce action from the singularities on the solution. According to [18,21], these are given by the entropy of the horizons. (See, for example, appendix of [25].)

$$
\begin{aligned}
I_{\mathcal{B}} & =-\frac{1}{16 \pi G_{5}} \int_{\mathcal{B}} R-\int_{\mathcal{B}} \mathcal{L}_{m}+\frac{1}{8 \pi G_{5}} \int_{\partial \mathcal{B}} K, \\
& =-\frac{1}{4 G_{5}} \sum_{i} \mathcal{A}_{i} .
\end{aligned}
$$

The bounce action $B$ for the decay process is given by subtraction of the action $I_{0}$ for the original configuration,

$$
B=I_{B}-I_{0}
$$

Finally, let us comment on a contribution to the bounce action from the singularity at the origin of the quintessence solution. For the solution with $-1 / 2<w \leq 0$ and $r_{\mathrm{BH}}=0$, there is an event horizon surrounding the origin, which allows us to consider only the outside region of it to calculate the bounce action. However, when we study the solution with $-1<w \leq-1 / 2$ and $r_{\mathrm{BH}}=0$, there is a 
naked singularity that can contribute to the bounce action. However, by using the method shown in [18], one can show that this singularity does not contribute. To show that, let us turn on $r_{\mathrm{BH}}$ and introduce a small black hole horizon, which can be regarded as a regulator for the calculation. In this case, by the method in [18], we find that its contribution is proportional to the area of the horizon. In the limit $r_{\mathrm{BH}} \rightarrow 0$, this vanishes. Therefore, we do not have to take into account the singularity at the origin.

\section{CATALYSIS INDUCED BY QUINTESSENCE}

In this section, we illustrate catalytic effects by discussing the freezing and thawing models separately. We investigate behavior of the bounce action as a functions of tension and quantities of the catalysts. We will find that in both cases, the bounce actions becomes smaller compared to that of Coleman-de Luccia, hence the lifetime of a metastable state is much shorter. We will see that there is a tendency that the catalytic effect becomes more important when $w$ approaches to $-1 / 2$.

\section{A. Catalysis in the freezing model}

As the first example of the catalysis, let us consider the freezing model: in the early stage of the Universe, $w$ is close to zero and gradually shifts to a smaller value (see Fig. 1). Thus, we consider catalysis induced by quintessence with
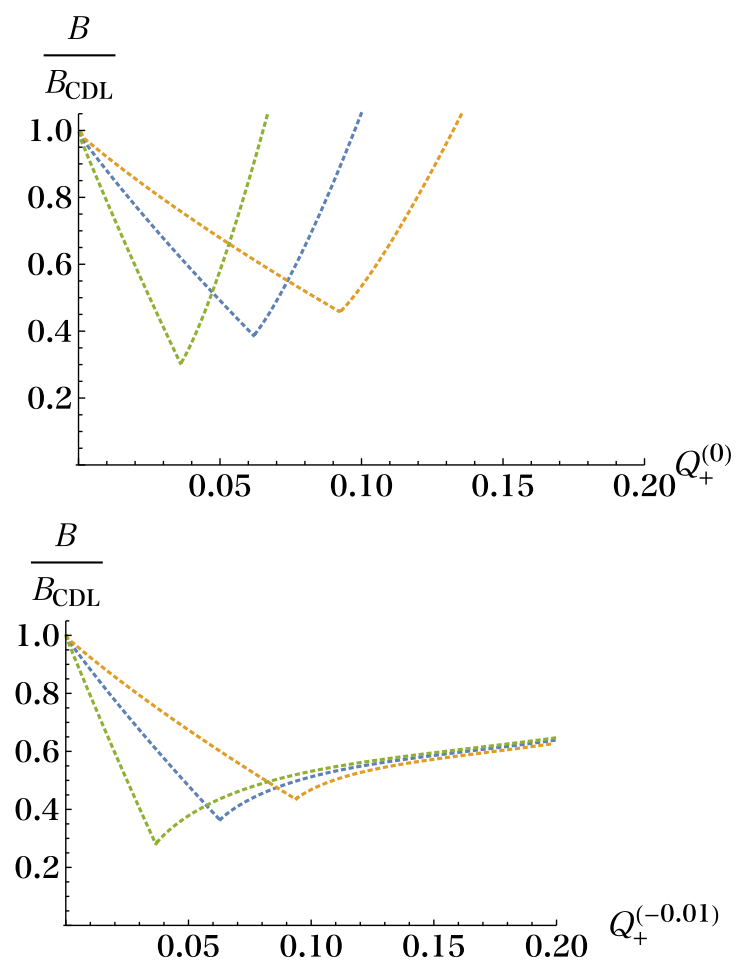

$w \sim 0$. We assume the decay of metastable Minkowski vacuum to AdS one. So, $f_{ \pm}$in this case are given by

$$
f_{+}=1-\frac{Q_{+}^{(w)}}{\tilde{r}^{4 w+2}}, \quad f_{-}=1-\frac{Q_{-}^{(w)}}{\tilde{r}^{4 w+2}}+\left(\frac{\gamma}{l_{-} \alpha}\right)^{2} \tilde{r}^{2}
$$

As mentioned in general arguments, the equation of motion for the bubble radius $\tilde{R}$ (in Euclidean time) is given by

$$
\begin{aligned}
(\dot{\tilde{R}})^{2}= & 1-\tilde{R}^{2}-\left(\bar{Q}+\frac{\Delta Q}{8 \eta^{2}}\right) \frac{1}{\tilde{R}^{4 w+2}} \\
& -\left(\frac{\left(1+4 \eta^{2}\right) \alpha \Delta Q}{16 \eta^{2}}\right)^{2} \frac{1}{\tilde{R}^{8 w+6}} .
\end{aligned}
$$

As an illustration, we show the bounce action for the cases, $w=0,-1 / 100,-1 / 10$, and $-3 / 10$ in Fig. 3. Remarkably, the catalytic effect is enhanced for small value of $w$. Therefore, in an explicit freezing model, the catalysis becomes more important as the time goes by. From the figure, we find that, for small value of $Q_{+}$, the bounce action is monotonically decreasing function and eventually reaches the minimum. We call the bubble at this point the saturated bubble. Above this minimum, the property of the bubble is changed and, in fact, there exists a remnant inside the bubble.

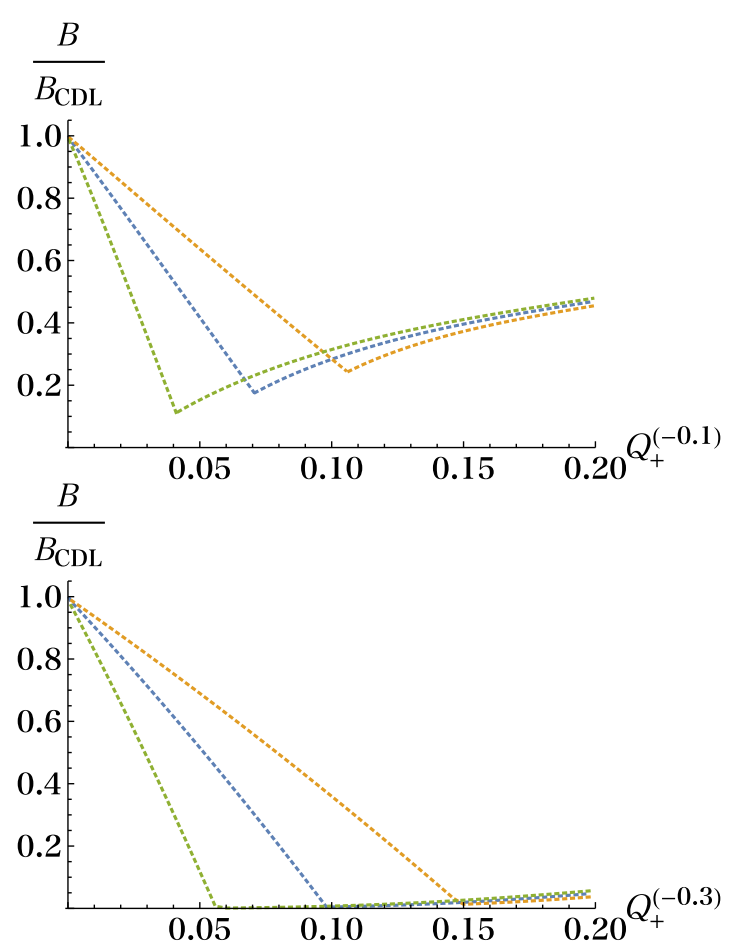

FIG. 3. The bounce actions for the quintessence with $w=0,-1 / 100,-1 / 10$, and $-3 / 10$. The green, blue, and orange curves correspond to $\eta=0.15,0.2$, and 0.25 . Beyond the minimum values of $Q_{+}^{(w)}$ for each curve, there is a remnant in the bubble. We refer to this bubble as the saturated bubble. 

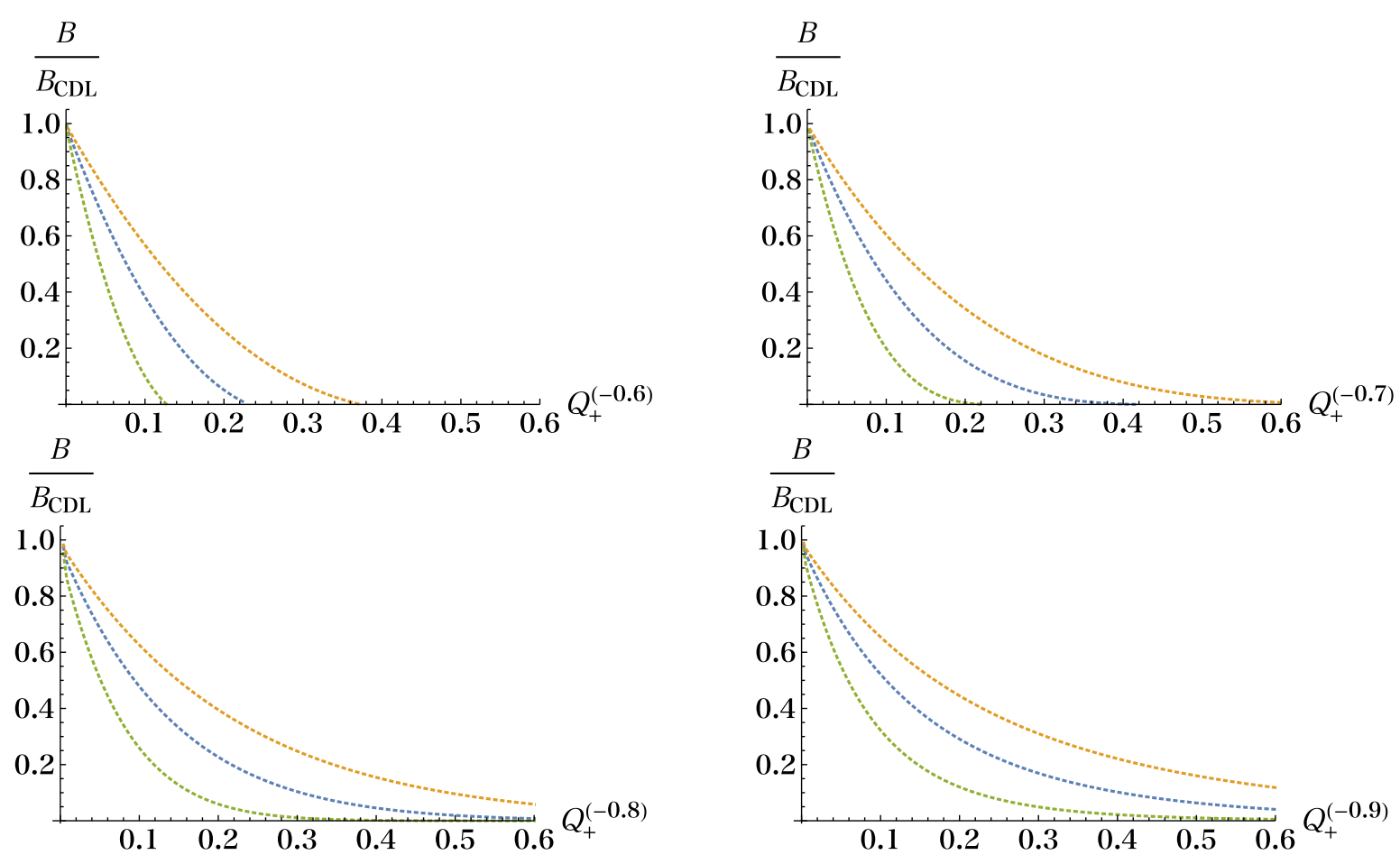

FIG. 4. The bounce actions for the thawing model with $w=-6 / 10,=-7 / 10,=-8 / 10$, and $=-9 / 10$. The green, blue, and orange curves correspond to $\eta=0.15, \eta=0.2$, and $\eta=0.25$.

What happen if we take a smaller value of $w$ ? The catalytic effect can become strong, however the inner horizon approaches to the size of the bubble since it is given by $\tilde{r}=Q_{+}^{1 /(4 w+2)}$. Clearly at $w=-1 / 2$, it diverges, which means that the horizon goes to infinity. This should be regard as a cosmological horizon. So with $w<-1 / 2$, the model is similar to the thawing type; we will discuss it in the next subsection.

\section{B. Catalysis in the thawing model}

As the second example of the catalysis caused by quintessence, let us study the thawing type behavior of $w$. In this model, at the early stage of the Universe $w$ is relatively close to -1 . In this subsection, we naïvely assume $-1<w<-1 / 2$. When $w \simeq-1$, the geometry becomes similar to the de Sitter spacetime because $f_{+}$ behaves like

$$
f_{+}=1-Q_{+} \tilde{R}^{a}, \quad a \simeq 2 .
$$

Again, we assume the metastable Minkowski vacuum, namely $\Lambda_{+}^{(5)}$ is zero, as an initial state. Since this $Q_{+}$plays a similar role to the cosmological constant of the de Sitter space, we find that smaller values of $Q_{+}$are energetically favorable. Thus, we expect that catalytic effect works in this model as well. Similarly, the geometry inside the bubble can be given by

$$
f_{-}=1-Q_{-} \tilde{R}^{a}+\left(\frac{\gamma}{l_{-} \alpha}\right)^{2} \tilde{R}^{2}
$$

As mentioned above, smaller $Q_{-}$is energetically favorable. The equation of motion for the bubble is given by (3.2). As an illustration, we show the bounce action for the model with $w=-6 / 10,=-7 / 10,=-8 / 10$, and $=-9 / 10$ in Fig. 4. Again, one finds that the bounce action becomes smaller as the parameter $w$ approaches to $-1 / 2$.

\section{REALIZATION OF INFLATION AND DARK ENERGY ON THE BUBBLE}

In the previous section, we studied the catalysis induced by quintessence. Both freezing and thawing models had an enhanced decay rate of metastable vacuum and quintessence played a role of catalyst. In this section, exploiting this understanding, we propose a model realizing inflation and the dark energy on the expanding bubble universe. On this bubble four-dimensional gravity is localized [10] due to AdS spaces inside and outside of the bubble. The cosmological constant for the four-dimensional theory can be determined by the tension of the bubble and vacuum energies of AdS spaces. Since the tension of bubble is fixed by the dynamics of the decay process, we can find most probable cosmological constant realized on this decay process. We will search in a wide range of parameter spaces of the theory. Then, we impose the trans-Planckian censorship conjecture (TCC) [26] and discuss the allowed cosmological constant, which gives us a constraint for the 
allowed parameter spaces. Since the created bubble itself is stable, as long as the lower energy vacuum is the absolute minimum, all the bubble with positive cosmological constant cannot satisfy TCC. This constraint gives us a remarkable scenario for realizing four-dimensional theory in string theories.

\section{A. Catalytic selection of bubble universe}

Let us include freezing and thawing types of quintessence simultaneously which eventually play roles of the dark energy and inflaton. Then, we assume that they have $w_{1} \simeq-1$ and $w_{2} \simeq 0$ when a bubble is created. Note that the most economical earlier example is the one discussed in $[31,32]$ where the single scale field plays not only the inflaton for the primordial inflation but also the quintessential dark energy in the late stage. However, this model cannot satisfy the conditions coming from the distance conjecture [7] and the trans-Planckian censorship conjecture [26], so we introduce two kinds of quintessence fields and consider a model circumventing the swampland conjectures. In the same way as the previous section, we study the decay of Minkowski vacuum to the AdS vacuum for the sake of simplicity. The inside and outside geometries that we will study are as follows:

$$
\begin{aligned}
& f_{+}=1-\frac{Q_{+(1)}^{\left(w_{1}\right)}}{\tilde{r}^{4 w_{1}+2}}-\frac{Q_{+(2)}^{\left(w_{2}\right)}}{\tilde{r}^{4 w_{2}+2}}, \\
& f_{-}=1-\frac{Q_{-(1)}^{\left(w_{1}\right)}}{\tilde{r}^{4 w_{1}+2}}-\frac{Q_{-(2)}^{\left(w_{2}\right)}}{\tilde{r}^{4 w_{2}+2}}+\left(\frac{\gamma}{l_{-} \alpha}\right)^{2} \tilde{r}^{2} .
\end{aligned}
$$

The equation of motion for the bubble connecting two geometries at $\tilde{r}=\tilde{R}$ is given by

$$
\begin{aligned}
(\dot{\tilde{R}})^{2}= & 1-\tilde{R}^{2}-\left(\bar{Q}_{(1)}+\frac{\Delta Q_{(1)}}{8 \eta^{2}}\right) \frac{1}{\tilde{R}^{4 w_{1}+2}} \\
& -\left(\bar{Q}_{(2)}+\frac{\Delta Q_{(2)}}{8 \eta^{2}}\right) \frac{1}{\tilde{R}^{4 w_{2}+2}} \\
& -\frac{1}{16 \eta^{2}}\left(\frac{l \alpha}{\gamma}\right)^{2}\left(\frac{\Delta Q_{(1)}}{\tilde{R}^{4 w_{1}+3}}+\frac{\Delta Q_{(2)}}{\tilde{R}^{4 w_{2}+3}}\right)^{2} .
\end{aligned}
$$

The original geometry before bubble nucleation has two horizons. In Fig. 5, we show an example of inner and outer horizons for the parameter choice, $w_{1}=-1 / 10$, $w_{2}=-7 / 10$, and $Q_{+(1)}^{(-0.1)}=3 / 100$. For fixed $Q_{+(1)}^{\left(w_{1}\right)}$ and $w_{1,2}$, there is the maximum value of $Q_{+2}^{\left(w_{2}\right)}$ where two horizons coincide. In the figure, it exists around $Q_{+2}^{(-0.7)} \sim 2.2$.

Now, we are ready to calculate the bounce action for the decay of the Minkowski vacuum to the AdS vacuum catalyzed by two quintessence fields. Especially, we focus on the following parameter choice, $\eta=0.2$,

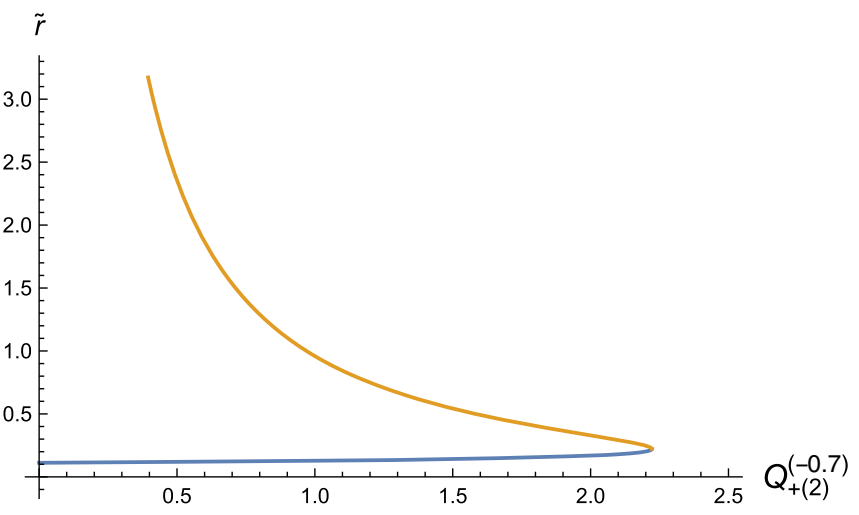

FIG. 5. Outer (the upper curve) and inner (the lower curve) horizons of the geometry before the transition. We choose $w_{1}=-1 / 10, \quad w_{2}=-7 / 10, \quad$ and $Q_{+(1)}^{(-0.1)}=3 / 100$. Around $Q_{+2}^{(-0.7)} \sim 2.2$ they coincide with each other.

$Q_{+(1)}^{(-0.1)}=3 / 100, \quad w_{1}=-1 / 10$, and $w_{2}=-7 / 10$. We show the ratio of the bounce action to that of CDL for various choices of remnants $Q_{-(1,2)}^{\left(w_{1,2}\right)}$ in Fig. 6. For a small value of $Q_{+(2)}^{(-0.7)}$, the dominant decay process is given by $Q_{-(1)}^{(-0.1)}=3 / 100$ and $Q_{-(2)}^{(-0.7)}=0$ (the purple curve), which means that the bubble with the remnant of quintessence of $w_{1}$ is the most probable possibility. On the other hand, when $Q_{+(2)}^{(-0.7)}$ is larger than about 0.04, the bubble without remnants, namely $Q_{-(1)}^{(-0.1)}=0$ and $Q_{-(2)}^{(-0.7)}=0$, dominate

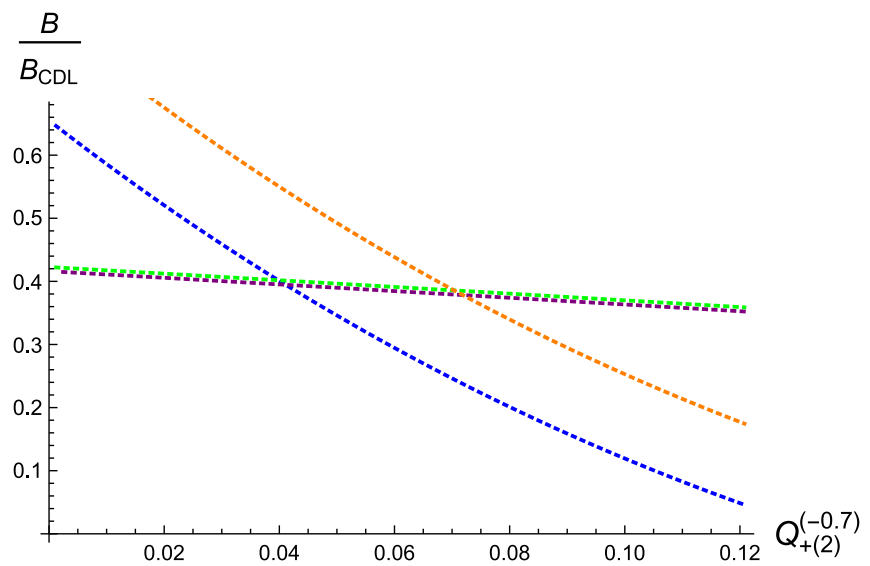

FIG. 6. The numerical calculation of the bounce action for the parameter choice $\eta=0.2, \quad Q_{+(1)}^{(-0.1)}=3 / 100, \quad w_{1}=$ $-1 / 10$, and $w_{2}=-7 / 10$. The blue curve corresponds to the bubble without remnant, namely $Q_{-(1)}^{(-0.1)}=0$ and $Q_{-(2)}^{(-0.7)}=0$. The orange and green curves have two types remnants, $Q_{-(1)}^{(-0.1)}=1 / 100, Q_{-(2)}^{(-0.7)}=1 / 100, Q_{-(1)}^{(-0.1)}=3 / 100$, $Q_{-(2)}^{(-0.7)}=1 / 100$, respectively. The purple curve includes only one remnant $Q_{-(1)}^{(-0.1)}=3 / 100, Q_{-(2)}^{(-0.7)}=0$. 

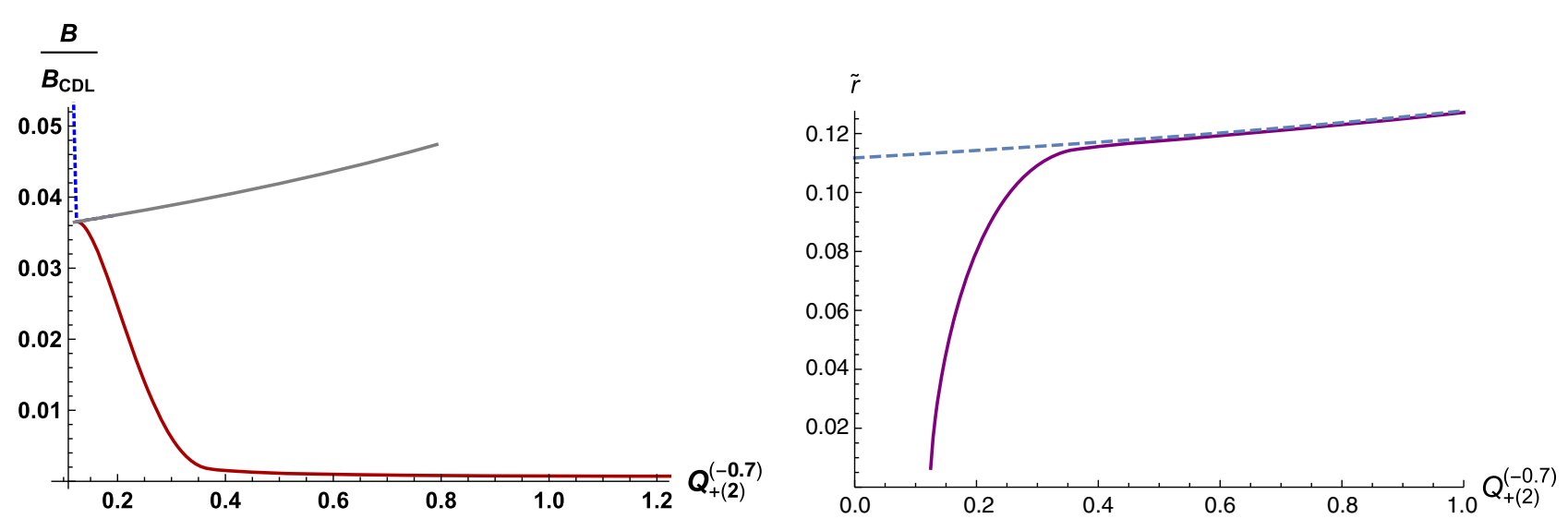

FIG. 7. Left panel: the bounce actions for the saturated bubble in which there is a remnant. We took $\eta=2 / 10, Q_{+(1)}^{(-0.1)}=3 / 100$, $w_{1}=-1 / 10$, and $w_{2}=-7 / 10$. The gray curve corresponds to the bubble with $Q_{-(1)}^{(-0.1)}=0$ and nonzero $Q_{-(2)}^{(-0.7)}$ while the brown curve corresponds to that with $Q_{-(2)}^{(-0.7)}=0$ and nonzero $Q_{-(1)}^{(-0.1)}$. The blue curve are connected to the end point of the one in Fig. 6. Right panel: purple curve is the position of the inner horizon inside the bubble. The doted blue line is that of original geometry before the transition.

the process (the blue curve). The former region, the catalytic effect induced by quintessence of $w_{2}$, is dominant while the latter is that of $w_{1}$. Since $w_{2}$ is smaller than $-1 / 2$, it behaves like positive cosmological constant which clearly enhances the decay rate of the vacuum in any region. On the other hand, as for $Q_{+(1)}^{\left(w_{1}\right)}$, the behavior is slightly different. Turning on $Q_{+(1)}^{(-0.1)}$ makes the event horizon in the geometry, which creates a singularity of the bounce solution. This enlarges the bounce action and forbids the decay of $w_{1}$ quintessence. However, by taking a large $Q_{+(2)}$, the catalytic effect overcomes that and allows us to decay the quintessence in the region of a large value of $Q_{+(2)}$.

Beyond $Q_{+(2)}^{(-0.7)} \sim 0.12$, the dominant contribution is given by the saturated bubble discussed in the previous section. In other words, the remnant has to be required to satisfy the equation of motion. The remnant should be $Q_{-(1)}^{w_{1}}$ or $Q_{-(2)}$. To see which remnant yields the dominant contribution, in the Fig. 7, we show two bounce actions corresponding to two choices of remnants for the parameter choice, $\quad \eta=2 / 10, \quad w_{1}=-1 / 10, \quad w_{2}=-7 / 10, \quad$ and $Q_{+(1)}^{(-0.1)}=3 / 100$. For the gray curve, we assumed $Q_{-(1)}^{(-0.1)}=$ 0 and nonzero $Q_{-(2)}^{(-0.7)}$. The nonzero value is determined by solving conditions for the saturated bubble. On the other hand, the blue curve is the opposite choice with $Q_{-(2)}^{(-0.7)}=0$ and nonzero $Q_{-(1)}^{(-0.1)}$. Clearly, the bounce action for the blue curve is smaller than the other.

Let us comment on the behavior of the blue curve around $Q_{+(2)}^{(-0.7)} \sim 0.3$. This drastic change of the behavior can be understood from the contribution of the inner horizon after the transition. As one can see in the right panel of Fig. 7, around $Q_{+(2)}^{(-0.7)} \sim 0.3$, the inner horizon after the transition approaches to the size of the horizon before the transition. Thus, the contributions from the singular part of the bounce solution before and after the transition becomes almost the same, which reduces the bounce action.

As we have shown in Figs. 6 and 7, the bounce actions become smaller as the parameter $Q_{+(2)}^{(w)}$ increases, which naïvely suggests to us that the most probable bubble corresponds to that of the smaller action because the decay rate is proportional to the action $\Gamma \sim e^{-B}$. However when $B$ is very small, the prefactor coming from one-loop contribution around the bounce solution can have a large contribution. By taking into account them, the lifetime of the vacuum is given by

$$
\tau \simeq\left(\frac{2 \pi}{B}\right)^{\frac{1}{2}} R_{B} e^{B},
$$

where $R_{B}$ is the size of the bubble. For numerical simulation it is useful to define dimensionless lifetime as $\tilde{\tau}=\alpha \tau / \gamma$. In Figs. 8 and 9 we show the bounce action and the lifetime of metastable vacuum as functions of $Q_{ \pm(2)}^{(w)}$ for the parameter choice, $Q_{+(1)}^{(-0.1)}=3 / 100, w_{1}=-1 / 10$, $w_{2}=-7 / 10, l^{3} / G_{5}=50$ and $1 / 100$. From the figures, we see that the lifetime of the vacuum is much shorter than that of the Coleman-de Luccia one in five dimensions,

$$
\tilde{\tau}_{\mathrm{CDL}} \simeq\left(\frac{2 \pi}{B_{\mathrm{CDL}}}\right)^{5 / 2} \tilde{R}_{B} e^{B_{\mathrm{CDL}}}
$$

The numerical estimations of the lifetime of CDL solution are shown in Fig. 10. The most probable universe can depend basically on two parameters, $l^{3} / G_{5}$ and $Q_{+(2)}^{(w)}$. In general, when $l^{3} / G_{5}$ is small, there is a tendency that the higher tension bubble is selected by the catalysis. By taking 

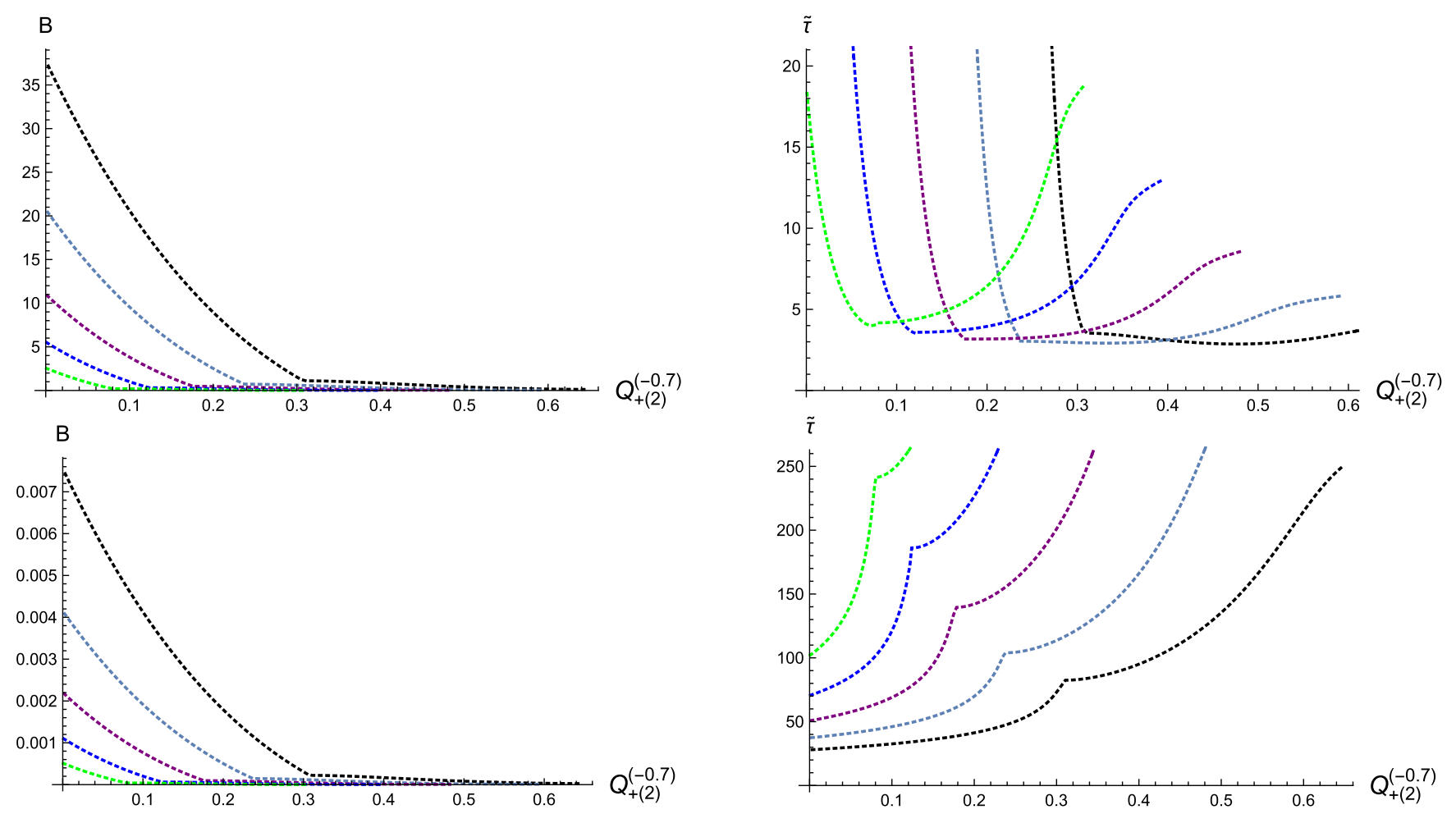

FIG. 8. We choose the parameters $Q_{+(1)}^{(-0.1)}=3 / 100, w_{1}=-1 / 10, w_{2}=-7 / 10$, and the green, blue, purple, light-blue, and black curves correspond to $\eta=0.18,0.2,0.22,0.24$, and 0.26 . In the upper two panels, we set $l^{3} / G_{5}=50$. In this case, the prefactor significantly contribute and bubbles with larger $B$ become the most probable decay. On the other hand, in the lower two panels, we set $l^{3} / G_{5}=1 / 100$. In this case, the values of the bounce action become larger compared to the previous case, the dominant contribution is given by the bubble with smaller bounce action.
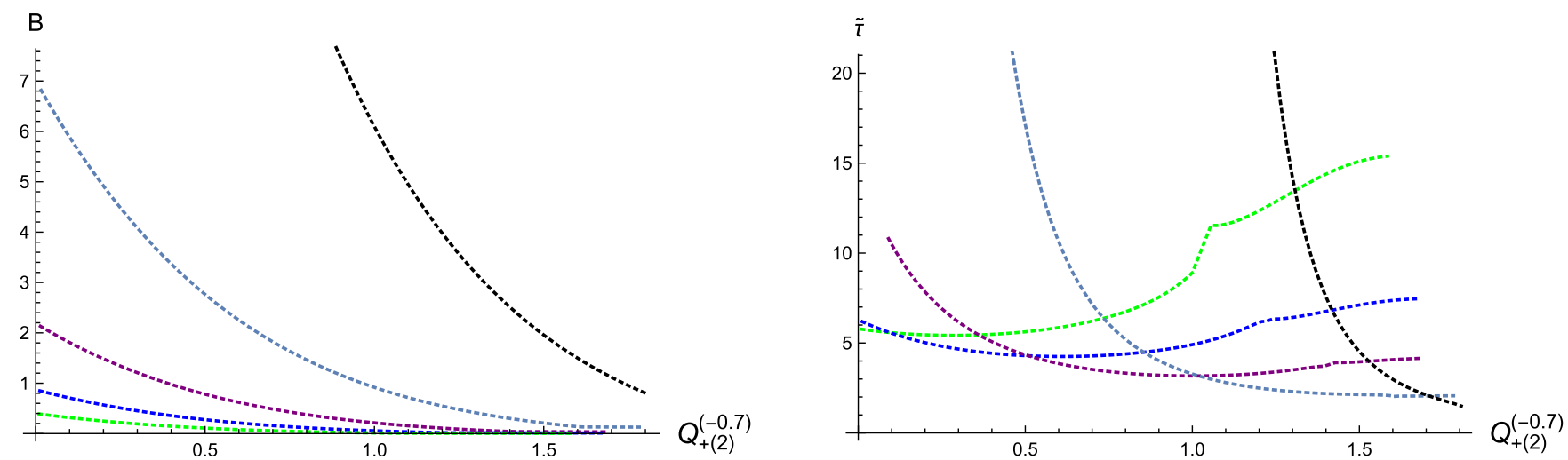

FIG. 9. The bounce actions for larger values of tension. The green, blue, purple, light-blue, and black curves correspond to $\eta=0.39$, 041, 0.43, 0.45, and 0.47. We took $l^{3} / G_{5}=1 / 100, Q_{+(1)}^{(-0.1)}=3 / 100, w_{1}=-1 / 10$, and $w_{2}=-7 / 10$.

$l^{3} / G_{5}$ to be very small, the tension of the most probable universe approaches to the critical tension (2.21). In this sense, for the critical bubble to have the largest probability by catalysis, we have to fine-tune the parameter $l^{3} / G_{5}$, which is the price we have to pay to get a very small cosmological constant on the four-dimensional bubble universe in light of the catalytic selection.

\section{B. Trans-Planckian censorship conjecture and catalytic creation of bubble}

In the previous subsection, we studied a selection of the bubble in terms of the catalysis and find that the cosmological constant on the bubble is determined by parameter choice of two vacua and $G_{5}$. Here, we discuss the selection from a slightly different viewpoint. In [26], 
$\log 10\left[\tilde{\tau}_{\mathrm{CDL}}\right]$

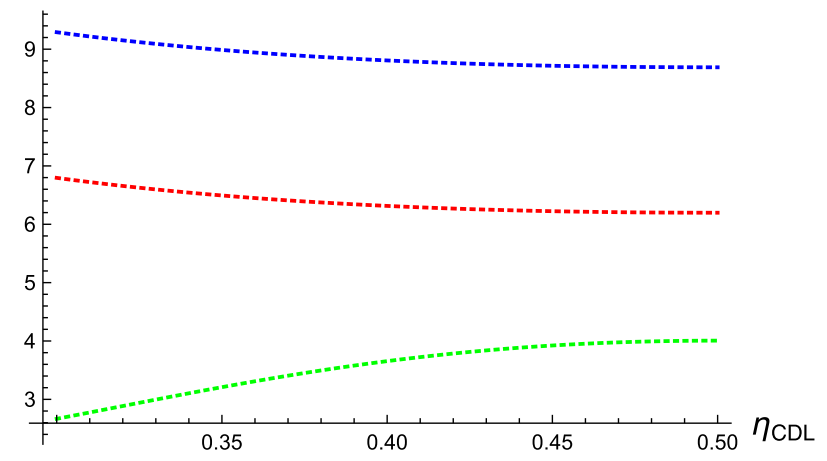

FIG. 10. Numerical estimation of lifetime for the CDL solution. Blue, red, and green curves correspond to $l^{3} / G_{5}=1 / 100,1 / 10$, and 50 .

the trans-Planckian censorship conjecture was proposed in the context of the swampland conjectures. Since we are interested in finding a scenario satisfying all conditions coming from a quantum theory of gravity, we would like to impose this condition to the bubble selection. TCC condition is given by

$$
\frac{R_{f}}{R_{i}}<\frac{M_{\mathrm{pl}}}{H_{f}}
$$

where $R$ is the scale factor of the four dimensions and subscripts $i$ and $f$ indicate the initial and final states. This condition yields a strong constraint for the lifetime of the de Sitter vacuum; thus, in our model, this should be imposed on the four-dimensional universe on the bubble. To avoid the trans-Planckian problem, the time period of the inflation, $T$, has to satisfy the following condition

$$
T<H^{-1} \log \left(\frac{M_{\mathrm{pl}}}{H}\right),
$$

where $H$ is the Hubble parameter during the inflation. In our setup, the created bubble exists eternally, as long as the lower energy vacuum is absolutely stable, it eventually violates the TCC condition. This is remarkable: although two vacua (in and outside) in the five dimensions are AdS spaces and satisfy all swampland conjectures including TCC, some of the created bubbles having the positive cosmological constant can violate the TCC condition. We interpret this fact as follows: since we naïvely assume that the tension of the bubble $\bar{\sigma}$ is a free parameter in the thinwall approximation that comes from the shape of the potential. However, in the low energy theory arising from a consistent theory of gravity, the allowed value of $\bar{\sigma}$ is limited, which indicates a constraint for the potential shape in AdS space. Hence, the bubble with a positive cosmological constant cannot be created under the decay process. Moreover, as mentioned in the previous sections, four-dimensional AdS space on the bubble also cannot be created by the decay process of metastable vacuum.
Namely, the bounce solution does not exist for this decay. Therefore, combining these two facts, we remarkably conclude that the created bubbles necessarily have vanishing cosmological constant. ${ }^{3}$

To see the most probable universe under this assumption, let us consider the equation of motion for the bubble

$$
\begin{aligned}
\left(\dot{\tilde{R}}^{\prime}\right)^{2}= & 1-\left(\bar{Q}_{(1)}^{\prime}+\frac{\Delta Q_{(1)}^{\prime}}{8 \eta^{2}}\right) \frac{1}{\tilde{R}^{\prime 4 w_{1}+2}} \\
& -\left(\bar{Q}_{(2)}^{\prime}+\frac{\Delta Q_{(2)}^{\prime}}{8 \eta^{2}}\right) \frac{1}{\tilde{R}^{\prime 4 w_{2}+2}} \\
& -\frac{1}{16 \eta^{2}}\left(\frac{l}{\gamma}\right)^{2}\left(\frac{\Delta Q_{(1)}^{\prime}}{\tilde{R}^{\prime 4 w_{1}+3}}+\frac{\Delta Q_{(2)}^{\prime}}{\tilde{R}^{\prime 4 w_{2}+3}}\right)^{2} .
\end{aligned}
$$

Note that the $\tilde{R}^{2}$ term does not exist in this case. Since we treat $\alpha=0$, it is convenient to redefine dimensionless parameters as follows:

$$
\begin{array}{ll}
\tilde{R}^{\prime}=\frac{R}{\gamma}, & \tilde{\lambda}^{\prime}=\frac{\lambda}{\gamma}, \\
\tilde{\tau}^{\prime}=\frac{\tau}{\gamma}, & Q_{ \pm(n)}^{(w)}=\left(\frac{1}{\gamma}\right)^{4 w+2} q_{ \pm(n)} .
\end{array}
$$

As in the previous subsection, we can calculate the bounce action and the lifetime of the metastable vacuum. Numerical estimations are shown in Fig. 11. Again, the lifetime is much shorter than that of CDL shown in (4.4).

\section{Application to cosmology}

Here, we will discuss expansion of the bubble after the nucleation by the catalysis. We use the Minkowski times and consider the evolution of the radius $R$. We focus on the critical bubble on which the cosmological constant is vanishing. As mentioned, the normalization of (2.13) is not appropriate since $\alpha=0$ and $\eta=1 / 2$, so we write the equation of motion in terms of dimensionful parameters

$$
\frac{\dot{R}^{2}}{R^{2}}=-\frac{1}{R^{2}}+\frac{q_{+(1)}}{R^{4 w_{1}+4}}+\frac{q_{+(2)}}{R^{4 w_{2}+4}}+\frac{l^{2}}{4}\left(\frac{\Delta q_{(1)}}{R^{4 w_{1}+4}}+\frac{\Delta q_{(2)}}{R^{4 w_{2}+4}}\right)^{2},
$$

(for critical bubble).

We use one of the quintessence fields in our model as an inflaton, namely the one corresponding to $w_{1}$. Right after the nucleation, it is still $w_{1} \simeq-1$ since the transition time is quite a short period. As long as $w_{1}$ is very close to -1 the inflation occurs and the radius of the bubble grows exponentially,

\footnotetext{
${ }^{3}$ Here, we assume that the lower energy vacuum with $\Lambda_{-}^{(5)}$ is absolute minimum. If it is metastable, then there is a chance to circumvent TCC condition by decaying into a lower energy vacuum.
} 

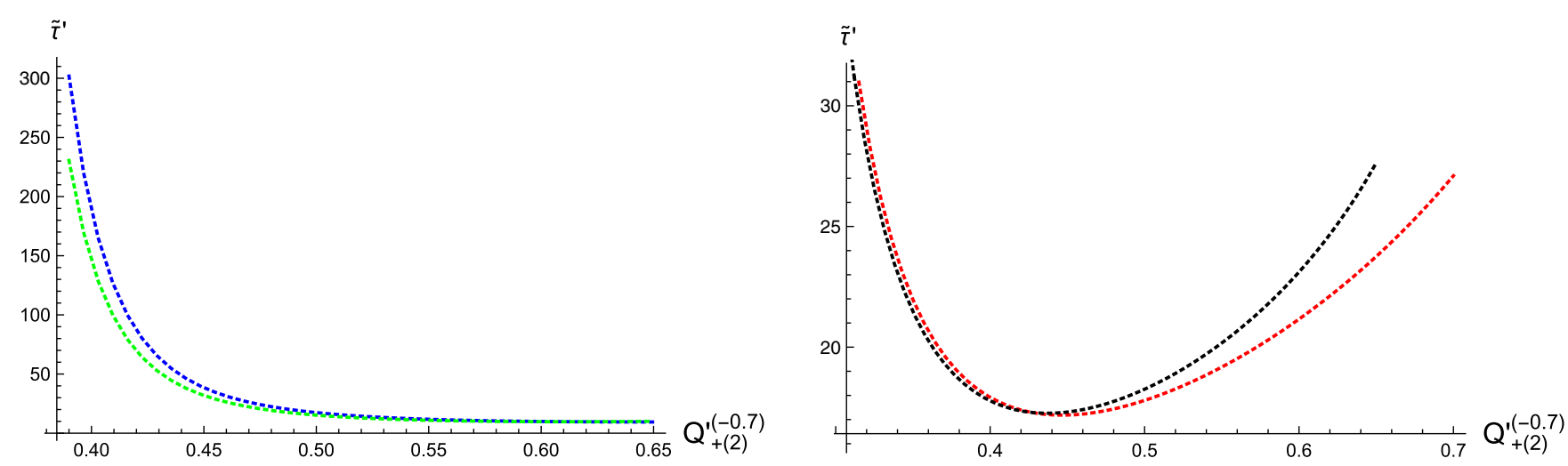

FIG. 11. Left panel: we choose $l^{3} / G_{5}=1 / 10, w_{1}=-1 / 10$, and $w_{2}=-7 / 10$. The blue and green curves correspond to $Q_{+(1)}^{(-0.1)}=$ $3 / 100$ and 1/10. Right panel: the red and black curves correspond to $Q_{+(1)}^{(-0.1)}=3 / 100$ and $1 / 10$. We took $l^{3} / G_{5}=1 / 100$, $w_{1}=-1 / 10$, and $w_{2}=-7 / 10$.

$$
R \propto \exp \left[\lambda \sqrt{q_{+(1)}}\right]
$$

For getting enough $e$-folding, e.g., $\mathcal{N}_{e} \sim 60$, we require an inflaton period $\lambda_{\text {inf }}$

$$
\lambda_{\text {inf }}=\mathcal{N}_{e} \frac{1}{\sqrt{q+(1)}}
$$

After this period, we assume that $w_{1}$ drastically varies its value and the relevant term in (4.9) becomes subdominant. When the term becomes comparable to other terms, the inflation stops.

After the inflation $R$ is exponentially large, thus most of the terms in (4.9) become irrelevant. This is nothing but the washout by inflation. However, since we assume the thawing type behavior for the second quintessence, $w_{2}$ eventually approaches to -1 . In this case, the following terms get back to relevant

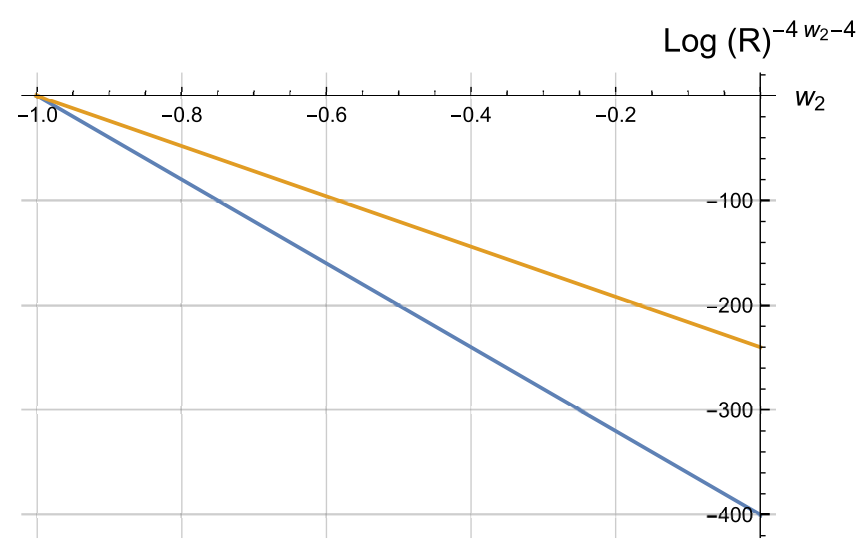

FIG. 12. The blue and orange lines correspond to $R=e^{100}$ and $e^{60}$. Near the region where $w_{2}$ is very close to zero, the functions $1 / R^{4 w_{2}+4}$ become drastically large.

$$
\frac{\dot{R}^{2}}{R^{2}}=\frac{q_{+(2)}}{R^{4 w_{2}+4}}+\frac{l^{2}}{4}\left(\frac{\Delta q_{(2)}}{R^{4 w_{2}+4}}\right)^{2}+\cdots
$$

As one can see in Fig. 12, a tiny value of function for general choice of $w_{2}$ is drastically enlarged as $w_{2}$ approaches to -1 . For example, the very small number $e^{-70}$ becomes large enough by their small powers, $\left[e^{-70}\right]^{\frac{1}{10}} \simeq 9.1 \times 10^{-4},\left[e^{-70}\right]^{\frac{1}{100}} \simeq 0.49$. This fact can be used to explain the smallness of the dark energy.

Finally, let us determine the quantity of the thawing quintessence in terms of data of present age as a boundary condition. We refer to the scale factor and $w_{2}$ at the present age as $R_{0}$ and $w_{0}$. For the quintessence to explain the small present dark energy, $q_{+(2)}$ should be

$$
\frac{\Lambda_{0}^{(4)}}{3} \simeq \frac{q_{+(2)}}{R_{0}^{4 w_{0}+4}} .
$$

\section{Toward a realistic model in five dimensions}

In our argument as far, we naively assumed the existence of two types of quintessential fields in five dimensions and discussed consequences of varying $w$ in time. It would be intriguing to explore a possibility of realization of our idea in an explicit model. One of the recent successful models for the quintessential inflation was studied in [33]. In the paper, the authors discussed an exponential type potential and studied the inflation at the early stage as well as the late-time dark energy in a single package. One of the interesting features of the paper is the scaling behavior of the inflaton field in the radiation dominated era. At the beginning of the era, the energy density of the inflaton becomes much smaller than that of the radiation. However, by the scaling behavior, which is peculiar property of the model, it gradually increases and approaches to the energy density of the radiation. This behavior is quite reminiscent to that of our model shown 
above (for example see Fig. 12). ${ }^{4}$ Therefore, it can be possible to realize an explicit model of our idea by slightly modifying it and using its scaling behavior. To do that, we first have to extend the model to five dimensions with a negative cosmological constant. Also, to make a metastable state, we have to assume the existence of a small dip at the origin of the inflaton field, for example. These modifications allow us to create the bubble discussed in this section. It would be interesting to explore this model further and study how these changes affect the scenario discussed in [33]. However, it is clearly beyond the scope of our paper, so we would like to revisit this issue in a separate publication.

This scaling behavior is also interesting because the energy density of the inflaton at the late stage does not depend on the initial value significantly. This idea also holds for our model shown in this section. The final values of cosmological constant in (4.13) does not highly depend on $q_{+}^{(2)}$ as long as it is nonzero. Dynamics of the late state that govern the behavior of $w_{2}$ is very important. In this sense, we can use the scaling mechanism to avoid the initial condition problem of the field corresponding to $w_{2}$.

\section{CONCLUSIONS AND DISCUSSIONS}

In this paper, we studied catalytic effects induced by quintessence in five dimensions. We computed the decay rate of a metastable vacuum by using Coleman's method and the technique to treat a singular bounce solution developed by [18]. We found that the decay rate is highly enhanced by the catalysis and the lifetime becomes much shorter. Since the lifetime varies from bubble to bubble, this can be seen as a dynamical selection of four-dimensional expanding universe. As in [10], we consider the critical bubble where the cosmological constant on the bubble is vanishing by tuning the parameters in five dimensions. This fine-tuning is a price we have to pay to get small dark energy. We also imposed the trans-Planckian censorship conjecture [26] on the decay process. The created bubble expands eternally, it eventually violates the condition, even if the cosmological constant is small. This contradicts with quantum gravity theory and can never occur in a consistent low energy theories. Moreover, since there is no solution corresponding to four-dimensional AdS spacetimes, we conclude that the only allowed bubble in a consistent theory of gravity has to have the vanishing cosmological constant.

In light of this understanding, we studied an application of this model to incorporate the inflation mechanism and the dark energy to the four-dimensional bubble universe. We introduced two types of quintessence fields, one is the thawing type that played a role of inflaton and the other is the freezing type that was used to explain the dark energy at

\footnotetext{
${ }^{4}$ We would like to thank the referee for showing us this model and suggesting the possibility of avoiding the initial condition problem by using this mechanism.
}

the present age. Initial inflation driven by the thawing-type field washes out the Universe; however, we claimed that the freezing type of quintessence can contribute at the late stage of the Universe since it can become $w_{2} \simeq-1$ and the very small number gets back to a large quantity. This idea may give us one of the possibility to explain the smallness of the dark energy.

In our setup, there is no contribution of matter and radiation at the late stage. To engineer them, we have to incorporate the reheating process in this scenario. It would be interesting to study the gravitational reheating process in the present quintessential inflation [31,34] and produce matter and radiation in this context, and study observational consequences along the lines of [32]. Since we naïvely assumed the time dependence of $w_{1,2}$ as shown in Fig. 1. The next step we should do is engineer an explicit model of quintessence in five dimensions and reproduce $w_{1,2}$ dependencies. Clearly, this is beyond the scope of our paper: we would like to revisit this issue in separate publication.

\section{ACKNOWLEDGMENTS}

The authors would like to thank Norihiro Tanahashi for useful discussions and Soichiro Hashiba for explaining to us quintessence inflation and his paper. We would like to thank the referee for pointing out the phantom field and an its possibility to our model. This work is supported by Grant-in-Aid for Scientific Research from the Ministry of Education, Culture, Sports, Science and Technology, Japan (Grants No. JP20K03932 and No. JP18H01214). The authors thank the Yukawa Institute for Theoretical Physics at Kyoto University, where the final stage of this work was done during the YITP-W-20-08 on "Progress in Particle Physics 2020.”

\section{APPENDIX A: COLEMAN-DE LUCCIA BOUNCE ACTION IN FIVE DIMENSIONS}

In this appendix, we will review the CDL bounce action $[4,14]$ by focusing on the five dimensions. The final expression will be used in the main text. We will adopt the same notations as the main text (2.13) and describe the position of the CDL bubble as $\tilde{r}=\tilde{R}(\tilde{\lambda}) . \tilde{\lambda}$ is the proper time on the bubble. For the tunneling between two vacua without quintessence, the equation of motion for the bubble reduces to

$$
\left(\frac{d \tilde{R}}{d \tilde{\lambda}}\right)^{2}=1-\tilde{R}^{2}
$$

The equation can be easily solved and the solution is given by $\tilde{R}=\cos \tilde{\lambda}$. The parameter range of the proper time is $-\pi / 2 \leq \tilde{\lambda} \leq \pi / 2$. By plugging back into the Euclidean action, we obtain the Coleman-de Luccia action 


$$
B_{\mathrm{CDL}}=\frac{\pi}{2 G_{5}}\left(\frac{\gamma}{\alpha}\right)^{3} \int_{-\frac{\pi}{2}}^{0} d \tilde{\lambda} \tilde{R}^{2}\left(\dot{\tilde{\tau}}_{+}-\dot{\tilde{\tau}}_{-}\right)
$$

where relations between the proper time and times of inside and outside geometries are described by

$$
\dot{\tilde{\tau}}_{ \pm}=\frac{1}{f_{ \pm}} \sqrt{f_{ \pm}-\left(\frac{d \tilde{R}}{d \tilde{\lambda}}\right)^{2}}=\frac{1}{f_{ \pm}} \sqrt{f_{ \pm}-\left(1-\tilde{R}^{2}\right)} .
$$

In this case, $f_{ \pm}$is simply represented as

$$
f_{ \pm}(R)=1-\kappa_{ \pm} \tilde{R}^{2}
$$

where $\kappa_{ \pm}$, which is a negative value for an AdS spacetime, is defined by

$$
\kappa_{+}=\left(\frac{\gamma}{l \alpha}\right)^{2}+\frac{\left(\alpha^{2}-1\right)}{\alpha^{2}}, \quad \kappa_{-}=\frac{\alpha^{2}-1}{\alpha^{2}} .
$$

Substituting this into (A3), we obtain

$$
\dot{\tilde{\tau}}_{ \pm}=\frac{\tilde{R}}{1-\kappa_{ \pm} \tilde{R}^{2}} \sqrt{1-\kappa_{ \pm}}
$$

Solving this equation, we find the relation between time in AdS and the proper time on the bubble. The result is

$$
t_{ \pm}=\frac{1}{\sqrt{\left|\kappa_{ \pm}\right|}} \tan ^{-1}\left(\frac{\sqrt{\left|\kappa_{ \pm}\right|}}{\sqrt{1+\left|\kappa_{ \pm}\right|}} \sinh \lambda\right),
$$

where we used the Minkowski time. As one can see from Fig. 13, even in the short period of the AdS spacetime, it corresponds to a long enough time on the bubble. Therefore, when we apply the trans-Planckian censorship conjecture [26] to the four-dimensional universe on the bubble, we have to use this proper time.

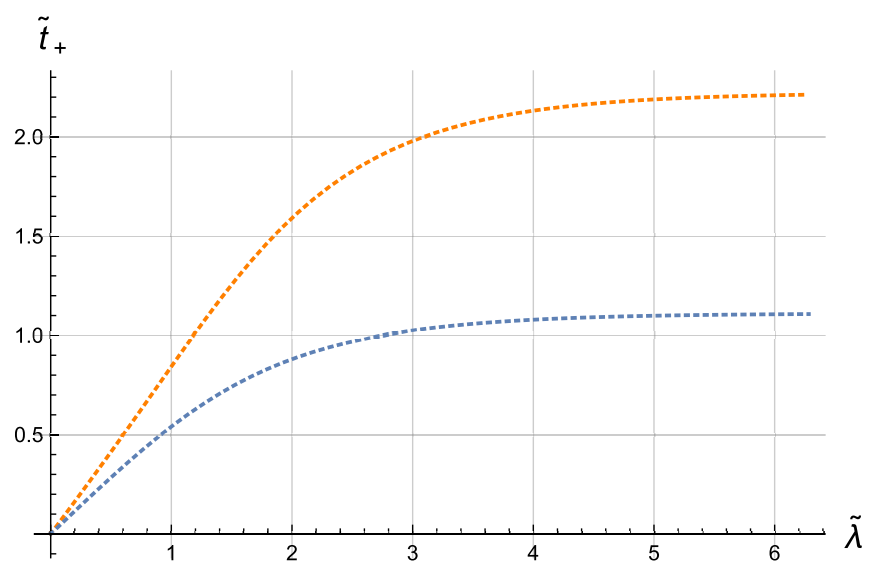

FIG. 13. The relation between the time $\tilde{t}_{+}$in AdS spacetime and the proper time $\tilde{\lambda}$ on the bubble. The blue and orange curves correspond to parameters $\kappa_{+}=-2$ and $\kappa_{+}=-1 / 2$, respectively.
Finally, we show the bounce action for the five dimensions in terms of an analytic function. We assume the decay of AdS (or Minkowski) to AdS vacua, namely, $\kappa_{ \pm} \leq 0$. In this case, the bounce action can be described in a relatively simple form:

$B_{\mathrm{CDL}}=\frac{\pi \gamma^{3}}{2 G_{5} \alpha^{3}}\left[\sqrt{1-\kappa_{+}} H\left(\kappa_{+}\right)-\sqrt{1-\kappa_{-}} H\left(\kappa_{-}\right)\right]$,

where we defined

$$
\begin{aligned}
H(\kappa) & \equiv \int_{0}^{1} d x \frac{x^{3}}{\left(1-\kappa x^{2}\right) \sqrt{1-x^{2}}} \\
& =\frac{1}{|\kappa|}-\frac{1}{\sqrt{|\kappa|^{3}(1+|\kappa|)}} \sinh ^{-1} \sqrt{|\kappa|} .
\end{aligned}
$$

\section{APPENDIX B: CATALYSIS BY PHANTOM FIELDS}

In the main text, we studied the catalysis induced by quintessential fields with $-1<w<0$. There is another interesting field, which is not excluded by observations, so-called the phantom fields with $w<-1$. This field plays an important role at the late stage of the Universe (see [35] for a review article and references therein). In this appendix, we investigate how the phantom field affects the catalysis at the early stage. To do that, we include a term in (2.5) corresponding to $w<-1$ and consider the junction condition for combining two different geometries. Doing in the same way as we did in Sec. III, we can calculate the bounce action for the catalysis. In Fig. 14, we show numerical estimations of the action with various choices of parameters, $w=-1,-1.1$, and -1.2 for the case $\eta=2 / 10$. As $Q^{(w)}$ for the phantom field increases,

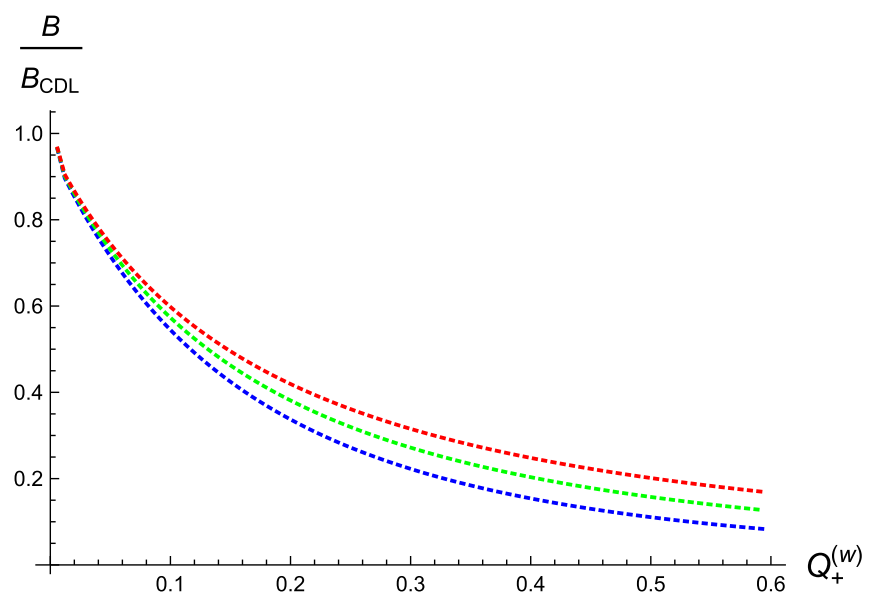

FIG. 14. The bounce action for the catalytic decays normalized by $B_{C D L}$ as a function of $Q_{+}^{(w)}$. We took $\eta=2 / 10$, and the blue, green, and red curves correspond to $w=-1,-1.1$, and -1.2 respectively. 
the value of the action decreases, which indicates enhancement of the decay rate of the metastable vacuum. However, as one can see from the numerical value of the vertical axis, the catalytic effect is mild compared with the case for $-1<w<0$. The reason for that can be seen in the form of $f(R)$; the contribution from the phantom field goes like $f=1-Q^{(w)} R^{a}$ where $a$ is greater than 2 . Hence, in the region of small $R$, the second term in $f$ becomes very small. Typically, the size of the created bubble is small, since it is a quantum effect, so the catalytic effect induced by the phantom field can be negligible compared to that of other fields. Although its effect is subdominant, the phantom field can play an important role at the late stage of the Universe such as the big rip. In this way, without disturbing the catalytic creation of the bubble universe at the early stage, we can include the phantom field and change the late time scenario drastically, which is an interesting possibility to explore further. Since this is beyond the scope of this paper, we would like to leave it for future work.
[1] G. Degrassi, S. Di Vita, J. Elias-Miro, J. R. Espinosa, G. F. Giudice, G. Isidori, and A. Strumia, J. High Energy Phys. 08 (2012) 098; A. Gorsky, A. Mironov, A. Morozov, and T. N. Tomaras, J. Exp. Theor. Phys. 120, 344 (2015); F. Bezrukov and M. Shaposhnikov, J. Exp. Theor. Phys. 120, 335 (2015); J. Ellis, J. Phys. Conf. Ser. 631, 012001 (2015); K. Blum, R. T. D’Agnolo, and J. Fan, J. High Energy Phys. 03 (2015) 166.

[2] M. S. Turner and F. Wilczek, Nature (London) 298, 633 (1982); I. Y. Kobzarev, L. B. Okun, and M. B. Voloshin, Sov. J. Nucl. Phys. 20, 644 (1975); M. Stone, Phys. Rev. D 14, 3568 (1976); P. H. Frampton, Phys. Rev. D 15, 2922 (1977).

[3] S. R. Coleman, Phys. Rev. D 15, 2929 (1977); C. G. Callan, Jr. and S. R. Coleman, Phys. Rev. D 16, 1762 (1977).

[4] S. R. Coleman and F. De Luccia, Phys. Rev. D 21, 3305 (1980).

[5] S. Chigusa, T. Moroi, and Y. Shoji, Phys. Rev. Lett. 119, 211801 (2017); Phys. Rev. D 97, 116012 (2018); C. Han, S. Pi, and M. Sasaki, Phys. Lett. B 791, 314 (2019).

[6] R. Bousso and J. Polchinski, J. High Energy Phys. 06 (2000) 006; S. Kachru, R. Kallosh, A. D. Linde, and S. P. Trivedi, Phys. Rev. D 68, 046005 (2003); L. Susskind, arXiv:hep-th/ 0302219; S. Ashok and M. R. Douglas, J. High Energy Phys. 01 (2004) 060.

[7] C. Vafa, arXiv:hep-th/0509212; H. Ooguri and C. Vafa, Nucl. Phys. B766, 21 (2007); H. Ooguri and C. Vafa, Adv. Theor. Math. Phys. 21, 1787 (2017).

[8] G. Obied, H. Ooguri, L. Spodyneiko, and C. Vafa, arXiv:1806.08362; S. K. Garg and C. Krishnan, J. High Energy Phys. 11 (2019) 075; H. Ooguri, E. Palti, G. Shiu, and C. Vafa, Phys. Lett. B 788, 180 (2019); H. Murayama, M. Yamazaki, and T. T. Yanagida, J. High Energy Phys. 12 (2018) 032.

[9] E. Palti, Fortschr. Phys. 67, 1900037 (2019); T. D. Brennan, F. Carta, and C. Vafa, Proc. Sci., TASI2017 (2017) 015 [arXiv:1711.00864]; U. H. Danielsson and T. Van Riet, Int. J. Mod. Phys. D 27, 1830007 (2018); Y. Akrami, R. Kallosh, A. Linde, and V. Vardanyan, Fortschr. Phys. 67, 1800075 (2019).

[10] S. Banerjee, U. Danielsson, G. Dibitetto, S. Giri, and M. Schillo, J. High Energy Phys. 10 (2019) 164; S. Banerjee, U. Danielsson, G. Dibitetto, S. Giri, and M. Schillo, Phys.
Rev. Lett. 121, 261301 (2018); S. Banerjee, U. Danielsson, and S. Giri, J. High Energy Phys. 04 (2020) 085.

[11] L. Randall and R. Sundrum, Phys. Rev. Lett. 83, 3370 (1999); Phys. Rev. Lett. 83, 4690 (1999).

[12] A. Karch and L. Randall, J. High Energy Phys. 09 (2020) 166.

[13] S. Banerjee, U. Danielsson, and S. Giri, J. High Energy Phys. 03 (2021) 250.

[14] I. Koga and Y. Ookouchi, J. High Energy Phys. 10 (2019) 281.

[15] P. J. Steinhardt, Nucl. Phys. B190, 583 (1981); Phys. Rev. D 24, 842 (1981); Y. Hosotani, Phys. Rev. D 27, 789 (1983); U. A. Yajnik, Phys. Rev. D 34, 1237 (1986).

[16] B. H. Lee, W. Lee, R. MacKenzie, M. B. Paranjape, U. A. Yajnik, and D. h. Yeom, Phys. Rev. D 88, 085031 (2013); B. Kumar, M. B. Paranjape, and U. A. Yajnik, Phys. Rev. D 82, 025022 (2010); B. Kumar and U. Yajnik, Nucl. Phys. B831, 162 (2010); B. Kumar and U. A. Yajnik, Phys. Rev. D 79, 065001 (2009); T. Hiramatsu, M. Eto, K. Kamada, T. Kobayashi, and Y. Ookouchi, J. High Energy Phys. 01 (2014) 165; K. Kamada, T. Kobayashi, K. Ohashi, and Y. Ookouchi, J. High Energy Phys. 05 (2013) 091; M. Eto, Y. Hamada, K. Kamada, T. Kobayashi, K. Ohashi, and Y. Ookouchi, J. High Energy Phys. 03 (2013) 159.

[17] A. Kasai and Y. Ookouchi, Phys. Rev. D 91, 126002 (2015); J. High Energy Phys. 06 (2015) 098; A. Kasai, Y. Nakai, and Y. Ookouchi, J. High Energy Phys. 06 (2016) 029; Y. Nakai and Y. Ookouchi, Phys. Lett. B 762, 321 (2016); Y. Nakai, Y. Ookouchi, and N. Tanahashi, Phys. Rev. D 100, 086013 (2019).

[18] R. Gregory, I. G. Moss, and B. Withers, J. High Energy Phys. 03 (2014) 081.

[19] W. A. Hiscock, Phys. Rev. D 35, 1161 (1987); V. A. Berezin, V. A. Kuzmin, and I. I. Tkachev, Phys. Lett. B 207, 397 (1988); I. G. Moss, Phys. Rev. D 32, 1333 (1985).

[20] N. Oshita and J. Yokoyama, Phys. Lett. B 785, 197 (2018); N. Oshita, M. Yamada, and M. Yamaguchi, Phys. Lett. B 791, 149 (2019); K. Kohri and H. Matsui, Phys. Rev. D 98, 123509 (2018); N. Oshita, K. Ueda, and M. Yamaguchi, J. High Energy Phys. 01 (2020) 015; K. Pasmatsiou, Phys. Rev. D 100, 125013 (2019); R. Gregory, I. G. Moss, N. Oshita, and S. Patrick, arXiv:2007.11428; T. Hayashi, K. Kamada, N. Oshita, and J. Yokoyama, J. High Energy Phys. 
08 (2020) 088; R. Gregory, I. G. Moss, and N. Oshita, J. High Energy Phys. 07 (2020) 024.

[21] P. Burda, R. Gregory, and I. Moss, J. High Energy Phys. 08 (2015) 114.

[22] P. Burda, R. Gregory, and I. Moss, J. High Energy Phys. 06 (2016) 025.

[23] L. Cuspinera, R. Gregory, K. Marshall, and I. G. Moss, Phys. Rev. D 99, 024046 (2019).

[24] L. Cuspinera, R. Gregory, K. M. Marshall, and I. G. Moss, Int. J. Mod. Phys. D 29, 2050005 (2020).

[25] I. Koga, S. Kuroyanagi, and Y. Ookouchi, Phys. Lett. B 800, 135093 (2020).

[26] A. Bedroya, M. Montero, C. Vafa, and I. Valenzuela, arXiv:2008.07555; A. Bedroya and C. Vafa, J. High Energy Phys. 09 (2020) 123; A. Bedroya, R. Brandenberger, M. Loverde, and C. Vafa, Phys. Rev. D 101, 103502 (2020).

[27] V. V. Kiselev, Classical Quantum Gravity 20, 1187 (2003).
[28] J. M. Toledo and V. B. Bezerra, Gen. Relativ. Gravit. 51, 41 (2019).

[29] E. J. Copeland, M. Sami, and S. Tsujikawa, Int. J. Mod. Phys. D 15, 1753 (2006); S. Tsujikawa, Classical Quantum Gravity 30, 214003 (2013).

[30] W. Israel, Nuovo Cimento Soc. Ital. Phys. B 44, 4349 (1966).

[31] P. J. E. Peebles and A. Vilenkin, Phys. Rev. D 59, 063505 (1999); M. Wali Hossain, R. Myrzakulov, M. Sami, and E. N. Saridakis, Int. J. Mod. Phys. D 24, 1530014 (2015).

[32] S. Hashiba and J. Yokoyama, Phys. Lett. B 798, 135024 (2019); S. Ahmad, A. De Felice, N. Jaman, S. Kuroyanagi, and M. Sami, Phys. Rev. D 100, 103525 (2019).

[33] C. Q. Geng, C. C. Lee, M. Sami, E. N. Saridakis, and A. A. Starobinsky, J. Cosmol. Astropart. Phys. 06 (2017) 011.

[34] L. H. Ford, Phys. Rev. D 35, 2955 (1987).

[35] K. J. Ludwick, Mod. Phys. Lett. A 32, 1730025 (2017). 